\title{
Pcl Injury in Children: Consequences of Being Neglected- A Case Report
}

\author{
Nishith Shah, Reetadyuti Mukhopadhyay*, Rohan Vakta, Jaymin Bhatt \\ Aash Arthtoscopy Centre, Ahmedabad, Gujarat, India
}

Received: February 22, 2016; Accepted: April 28, 2016; Published: May 07, 2016

*Corresponding author: Reetadyuti Mukhopadhyay, Aash Arthtoscopy Centre, Ahmedabad, Gujarat, India; E-mail: reetadyutim@gmail.com

\begin{abstract}
The diagnosis of an acute PCL injury is often missed. Thus, despite the excellent healing potential of PCL, an improperly or untreated patient develops symptoms of chronic PCL laxity. A knee with long term PCL laxity in turn is predisposed to early degenerative changes. We present the case of a young boy with neglected PCL injury of almost a year leading to posterior capsular contracture and severe morbidity. PCL augmentation was done in two stages. In the first stage posterior capsular release was done and double pin traction given. In the second stage a PCL augmentation was done. This is an excellent case to highlight the importance of diagnosing an acute PCL injury and managing is properly.
\end{abstract}

Keywords: PCL injury; Posterior Capsular Contracture; Paediatric PCL injury

\section{Introduction}

There is very little literature on the management of PCL injuries in children. These children are often brought late by their parents for repeated synovitis, effusion, medial compartment and parapatellar pain and difficulty in performing daily activities. . $^{1,2,3,4,5}$ The main complaint of all these children is difficulty in sitting cross-legged on the ground for a long time and to use Indian type of toilet that is prevalent in Indian society, requiring the patient to squat. These children refuse to play and complain of difficulty in climbing stairs.

PCL has a very good healing ability. A diagnosis is however often not made; particularly in the initial stagesand almost all of these acute PCL injuries go undiagnosed and untreated. Many children are able to compensate thelong termPCL laxity from a functional point of view. It is very difficult to say how many of them become symptomatic, ${ }^{6}$ however with increasing passage of time, symptoms and secondary changes start becoming apparent despite excellent muscle strength. ${ }^{7}$

We wish to report a 12 years old boy with a one year old neglected PCL injury leading to development of posterior capsular contracture and secondary changes in the knee joint.

\section{Case Report}

A previously active young boy of 12 years age presented to us complaining of pain in the right knee. He had difficulty in squatting and sitting cross-legged for almost 1 year. He could not run, and had stopped playing. His pain worsened on trying to play, run or climb stairs.

He gave history of having fallen while cycling a year back. His fully bent knee had his the ground and he had taken multiple treatments ever since, but not for once was a ligament laxity diagnosed.

Examining the child showed evident posterior tibial sag. (Figure 1) He had joint line tenderness but no effusion or synovitis. His range of motion was $0-110^{\circ}$ compared to $0-140^{\circ}$ on the opposite side. Despite the evident posterior tibial sag, the posterior drawer test did not show significant laxity. Based on the history and the clinical findings we reached the probable diagnosis of a chronic PCL laxity with development of posterior capsular contracture.

We asked the child to get a roentgenogram and MRI done. The X-ray confirmed the posterior tibial sag and the MRI (Figure 2) showed a stretched PCL with tear in the lateral meniscus along with cystic changes.

A two stage surgical treatment was planned and discussed with the child's parents. In the first stage, we did an arthroscopic posterior capsular release through the postero-medial portal and debrided the lateral meniscus tear (Figure 3), as it was

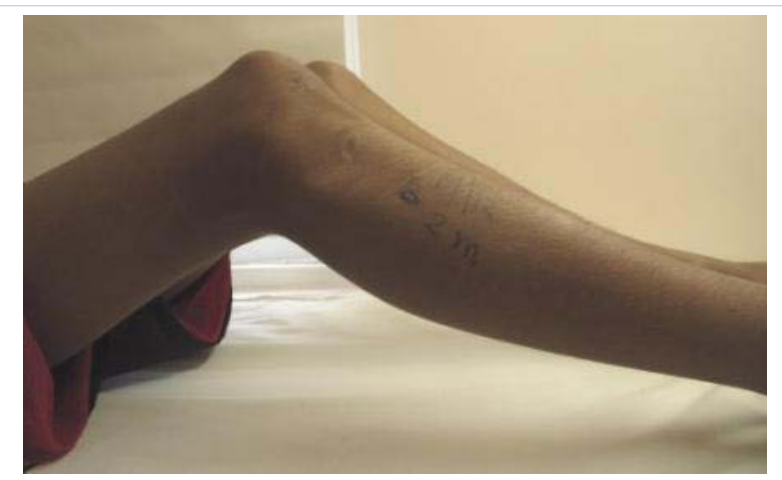

Figure 1: Clinical photo showing the evident posterior tibial sag on the right side. 


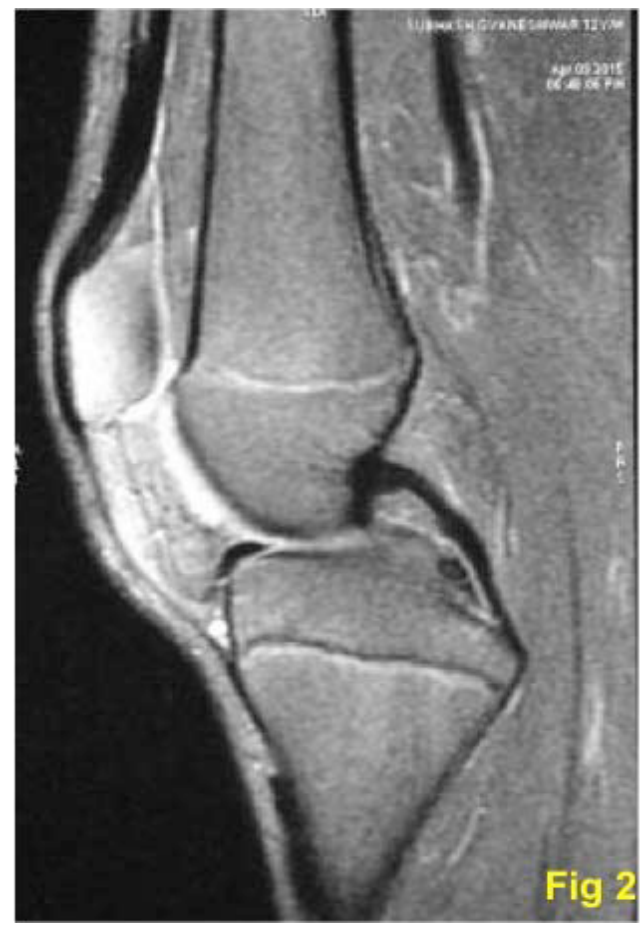

Figure 2: Arthroscopic picture of the complex lateral meniscus tear.

un-repairable. Post-operatively the child was given double pin traction with upper tibial and lower tibial steinmann pins for 3 weeks. (Figure 4)

The traction was removed after 3 weeks and the range of motion exercises were explained to the child while maintaining PCL brace. (Figure 5) He was re-examined after 1 month, when he had regained the pre-operative range of motion. He had no swelling or synovitis. PCL laxity grade II was evident. Both the lower limbs were of equal length and the height of the child was $162 \mathrm{cms}$.

In the second stage, about a month after removal of the traction and once the child had regained the pre-operative range of motion, a PCL reconstruction / augmentation surgery was planned. PCL augmentation was done using hamstring graft harvested from the same limb. Graft fixation was done using an Endobutton CL (Smith \& Nephew) of $15 \mathrm{~mm}$ length for the femoral side and a suture disc for the tibial side. (Figure 6)

The child was given a PCL brace post-operatively and kept non-weight bearing for 10 days. He was however allowed prone knee bending 5 days after surgery. The child was instructed to do straight leg raise 50-60 times a day along with quadriceps strengthening exercises from the $3^{\text {rd }}$ post-operative day. The PCL brace was removed after 6 weeks, and the child was given a knee-cap for day time and was asked to continue with the brace at night time for another 4-6 weeks.

Two months after PCL surgery, the boy came back history of slipping down the stairs at school. He sustained a hyperextension injury to the operated knee. He had attained $0-120^{\circ}$ of knee movement. On examining the child he had grade I PCL laxity. It was decided to give the child a trial of prolotherapy ${ }^{8}$.

The child was re-examined one month later. He had no complaints and was doing straight leg raise with $5 \mathrm{Kg}$ weight comfortably. The range of motion was $0-130^{\circ}$ with no PCL laxity.

\section{Discussion}

PCL is the most important stabilizers of the knee and is twiceas strong as the ACL. ${ }^{9}$ It prevents the posterior translation of the tibia on the femur. When the PCL is avulsed or stretched, the tibia moves posteriorly. The patient does not complain of instability unlike in ACL injury. Thus, patients with PCL injuries present late, and are often undiagnosed. ${ }^{9}$ The PCL may get injured by a direct impact, as occurred in our patient due to fall from the cycle, the flexed knee forcefully hit the ground pushing the tibia back on the femur and stretching the PCL. Hyperextension injury, as may occur due to slip from stairs can also result in a PCL tear. ${ }^{5}$

The excellent healing potential of PCL is a well-known fact. ${ }^{10}$

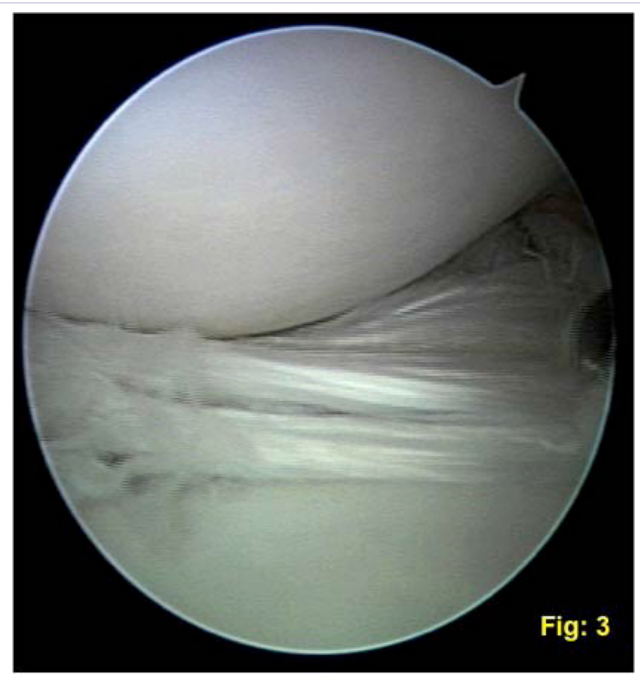

Figure 3: The child was put on double pin traction to correct the posterior capsular contracture after arthroscopic posterior capsular release.

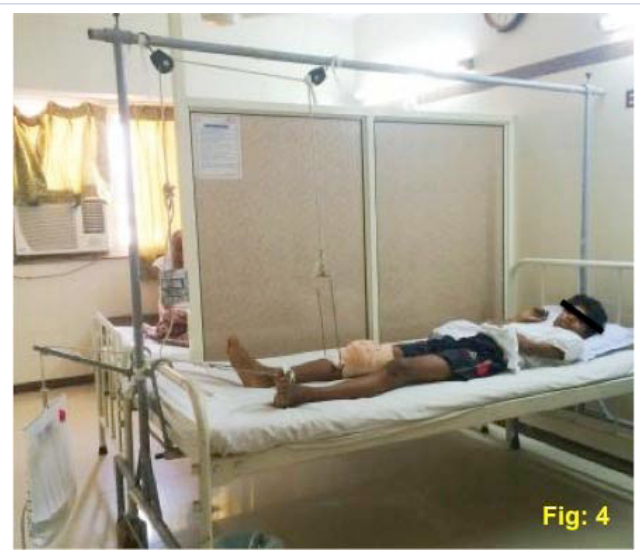

Figure 4: The child was given a custom made PCL brace post surgery and was instructed to do exercises while maintaining the brace. 


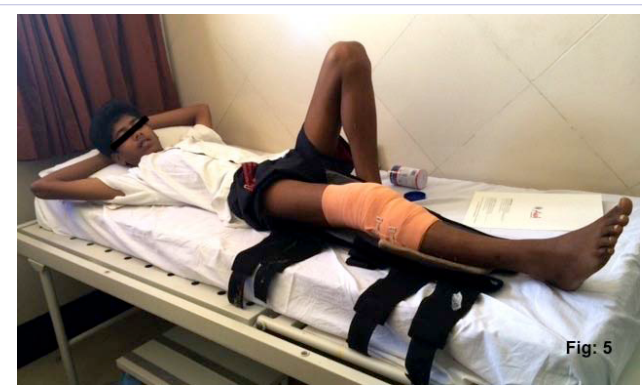

Figure 5: Radiograph post PCL surgery showing the physeal sparing tunnels and fixation of the graft on both sides.

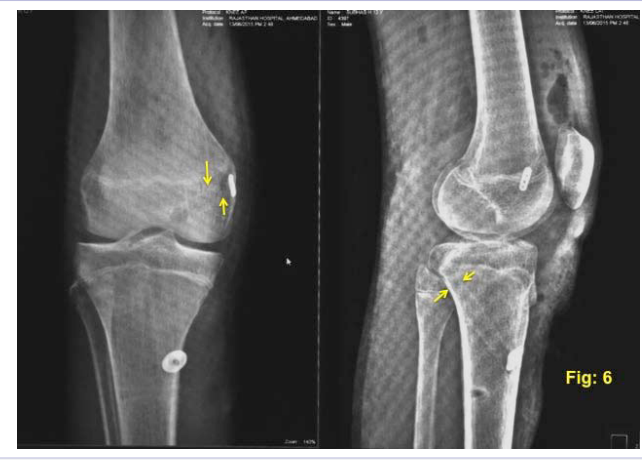

Figure 6

PCL injuries are however often not diagnosed in the acute stage. Such patients cope well with quadriceps strengthening and lifestyle modifications. However, over a period of time PCL lax patients develop symptoms of recurrent effusions and synovitis, difficulty in squatting and sitting cross-legged and early fatigue of calf leading to refusal to play. These complaints must not be overlooked and a proper diagnosis of PCL laxity must be made and managed with PCL reconstruction / augmentation. Such patients if neglected further develop degenerative changes in the knee, particularly patella-femoral arthritis and medial joint arthritis. $^{7}$

This case is an excellent example to highlight how often an acute PCL injury is missed and improperly treated, leading to secondary changes. It also highlights the consequences of a chronically neglected lax PCL leading to degenerative changes in the knee.

Had this child been properly diagnosed and treated at the first instance, he would have been spared the prolonged morbidity of almost a year and the burden of two surgeries. It is thus, very important to make a right diagnosis based on detailed history and a properly done physical examination.

It is also very important to follow a stringent conservative treatment protocol for acute PCL injuries with regular follow up and routine assessment of the improvement in PCL laxity grade. ${ }^{10}$

\section{Limitation}

We need a longer follow up until completion of growth to ensure no growth deformity or limb length disturbance occurs. Routine clinical as well as radiological follow up is required in this patient despite using physes sparing tunnels as is evident in figure 5 .

\section{References}

1. Warme WJ, Mickelson D. All-epiphyseal semitendinosus PCL reconstruction in a 10-year-old child. J Pediatr Orthop. 2010;30(5):465-468.

2. Accadbled F, Knörr J, Sales de Gauzy J. All inside transtibial arthroscopic posterior cruciate ligament reconstruction in skeletally immature: Surgical technique and a case report. Orthop Traumatol Surg Res. 2013;99(3):361-365. doi: 10.1016/j.otsr.2012.11.017.

3. Anderson AF, Anderson CN. Posterior cruciate and postero- lateral ligament reconstruction in an adolescent with open physes. A case report. J Bone Joint Surg Am. 2007;89(7): 1598-1604.

4. Bovid KM, Salata MJ, Vander Have KL, Sekiya JK. Arthroscopic posterior cruciate ligament reconstruction in a skeletally immature patient: a new technique with case report. Arthroscopy. 2010;26(4):563570. doi: 10.1016/j.arthro.2010.01.029.

5. Kim SJ, Jo SB, Kim SG, Park IS, Kim HP, Kim SH. Peel-off injury at the tibial attachment of the posterior cruciate ligament in children. Am J Sports Med. 2010; 38(9):1900-1906.

6. Kongcharoensombat W, Nakamae A, Adachi N, Deie M, Mohamed A, Sumen Y, et al. Mid- substance tear of the anterior and posterior cruciate ligaments in children: a report of three patients. Knee Surg Sports Trau- matol Arthrosc. 2009;17(8):964-967.

7. Keller PM, Shelbourne KD, McCarroll JR, Rettig A.C. Nonoperatively treated isolated posterior cruciate ligament injuries. Am J Sports Med. 1993; 21(1): 132-136.

8. Reeves, K. Dean, Khatab M. Hassanein. Long-term effects of dextrose prolotherapy for anterior cruciate ligament laxity. Alternative therapies in health and medicine. 2003; 9(3): 58-62.

9. Kocher MS, Shore B, Nasreddine AY, Heyworth BE. Treatment of posterior cruciate ligaments injuries in paediatric and adolescent patients. J Pediatric Orthopedics. 2012; 32(6):553-560.

10. Sanders WE, Wilkins KE, Neidre A. Acute insufficiency of the posterior cruciate ligament in children. Two case reports. J Bone Joint Surg Am. 1980;62(1):129-131.

11. Jones MT, Skidmore BL (2015) Effect of a 12-week Resistance-exercise Training Program on the Physical Self-perceptions of Children. J Exerc Sports Orthop 2(3): 1-6. 